\title{
MPPT ALGORITHM BASED ON PARTICLE SWARM OPTIMIZATION WITH NATURAL SELECTION
}

\author{
Yishuang $\mathrm{Hu}^{1, \mathrm{a}}$, Jingyi $\mathrm{Lu}^{1}$, Yue Deng ${ }^{1}$, Zimu Zhang ${ }^{1}$, \\ 1. School of electric power engineering, North China Electric Power University, \\ Baoding 071000, China; \\ 273415335@qq.com
}

Keywords: non-uniform light, particle swarm optimization, natural selection

\begin{abstract}
The output of photovoltaic array under non-uniform light is nonlinear with multi peaks. Since the traditional MPPT algorithm based on particle swarm optimization (PSO) is likely to fall into premature convergence and may work slower in the later iterations, the MPPT method based on improved PSO with natural selection was proposed for the first time in the paper. New algorithm worked by eliminating and replacing the particles with lower fitness during the iterative process and hence the swarm could track better and faster for the maximum point, with less chances of premature convergence. During the MATLAB modeling and simulation, the improved MPPT algorithm could perform better than the traditional PSO method.
\end{abstract}

\section{Introduction}

Currently, scholars have put forward some traditional methods of MPPT, like Perturbation and observation Method, Conductance Increment Method and Optimal Gradient Method. All of these methods work well in conditions of uniform illumination change and constant temperature. At present, main MPPT algorithms for complex lighting conditions in the world mainly consist of Particle Swarm optimization (PSO) ${ }^{[2][3]}$, Genetic Algorithms(GA) . Because of its simple and quick solving, Particle Swarm optimization is used the most widely. However, tradition PSO may cause premature convergence during the search. In particularly, traditional PSO is easy to fall into local extreme point in the condition of more output power peaks of PV array. Additionally, the performances of PSO will usually decline late in the search. Refer to the thought of Natural selection particle swarm optimization, the new method is proposed that combine the Natural selection mechanism with traditional MPPT to improve Particle Swarm to find the optimal solution, which is replacing the speed and place of the half particle whose fitness is the worst by the best in each iteration.

\section{Output characteristics of PV array}

PV arrays are often a mixture of a plurality of PV modules connected in parallel and series if the lighting conditions are uneven, the modules will produce different photocurrent and voltage.

For the array consisted of two photovoltaic modules 1 and 2 connected in series, assuming that module 1 has uniform illumination and module 2 is kept out. $I_{P H} 1$ and $I_{P H} 2$ are photocurrents of two components apartly, the output equation of the array is as follows:

$$
\begin{aligned}
& U=\frac{n K T}{q}\left[\ln \left(\frac{I_{P H 1}-I}{I_{0}}+1\right)+\ln \left(\frac{I_{P H 2}-I}{I_{0}}+1\right)\right]-2 I R_{S} \quad\left(0, I_{P H} 2\right) \\
& U=\frac{n K T}{q} \ln \left(\frac{I_{P H}-I}{I_{0}}+1\right)-I R_{S} \quad\left(\begin{array}{lll}
I_{P H} & 2
\end{array}, I_{P H} 1\right)
\end{aligned}
$$


Using MATLAB to simulate, the output characteristic of the array is shown as follows. As shown in the Fig.1, when the lighting condition is uneven, the output power of the PV array has a number of extreme points. Traditional MPPT algorithm may just find a local extreme point and cannot track the maximum power point.

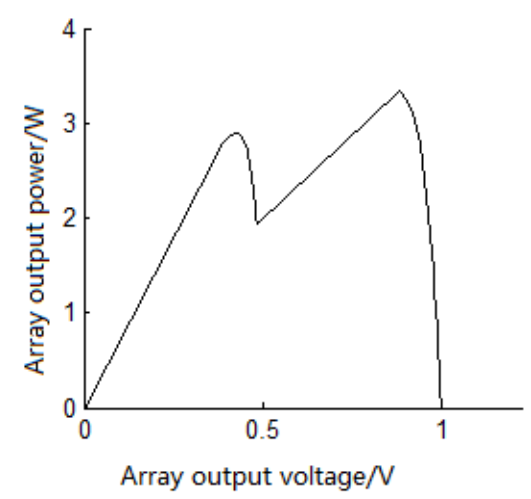

Fig.1 The output characteristic of series PV model under non uniform illumination

\section{The model of the maximum power point}

According to the mathematical model of the solar cell, the expressions of array output power in the case of connection are as follows:

$$
\begin{aligned}
& P=\sum_{i=1}^{2}\left\{\ln \left[\left(1-\frac{I}{I_{s c}}\right) \frac{1}{C_{1}}+1\right] \bullet C_{2} \bullet V_{o c}\right\} \bullet I \\
& C_{1}=\left(1-\frac{I_{m}}{I_{s c}}\right) \bullet \exp \left(-\frac{V_{m}}{C_{2} V_{o c}}\right) \quad C_{2}=\left(\frac{V_{m}}{V_{o c}}-1\right) \bullet\left[\ln \left(1-\frac{I_{m}}{I_{s c}}\right)\right]^{-1}
\end{aligned}
$$

Where $V_{m}$ and $I_{m}$ is the voltage and current corresponding to the maximum power point, $V_{o c}$ is the open-circuit voltage, $I_{s c}$ is the short circuit current of the module. It is abvious that the expression above is hard to sovle. In this article, the models above will be soveld using the improved PSO algorithm based on Natural Selection

\section{Improved Particle Swarm MPPT algorithm based on Natural Selection}

In the PSO algorithm, each particle $\mathrm{i}$ of $\mathrm{N}$ dimensional search space have a function to be optimized by a determined adaptation value (fitness value) and the velocity vector which can determine its flight direction and distance. Meanwhile, all particles know the best position found by itself (Personal best) and the best position found by currently groups (Global optimal solution). The update formulas of position and velocity are followed. Where $\mathrm{w}$ is the inertia weight factor, $c_{1}$ and $c_{2}$ are positive learning factors, $r_{1}$ and $r_{2}$ are random numbers uniformly distributed between 0 and $1, \mathrm{~d}$ is the spatial dimensions, $p_{i, j}$ is the Pbest, and $p_{g, j}$ is the global optimal solution.

$v_{i, j}^{t+1}=w v_{i, j}^{t}+c_{1} r_{1}\left(p_{i, j}-x_{i, j}^{t}\right)+c_{2} r_{2}\left(p_{g, j}-x_{i, j}^{t}\right) \quad x_{i, j}^{t+1}=x_{i, j}^{t}+v_{i, j}^{t+1}, j=1,2, \ldots d$

The idea to improve algorithm are adding new programs and algorithms in the late particle swarm iterations to improve its post-optimization capability and better out of local minima. Combine the natural selection with traditional particle swarm MPPT algorithm, the core idea of which is to replace 
the half of the particles with poorer fitness value with the other half with better fitness value in every iterative process, and retain the optimal solution of each particle's historical memory at the same time. Thus, particle population makes evolution and replacement continuously in the process of MPPT, which effectively reduce the likelihood of falling into premature convergence, while improving the search speed and optimization accuracy of the particle populations.

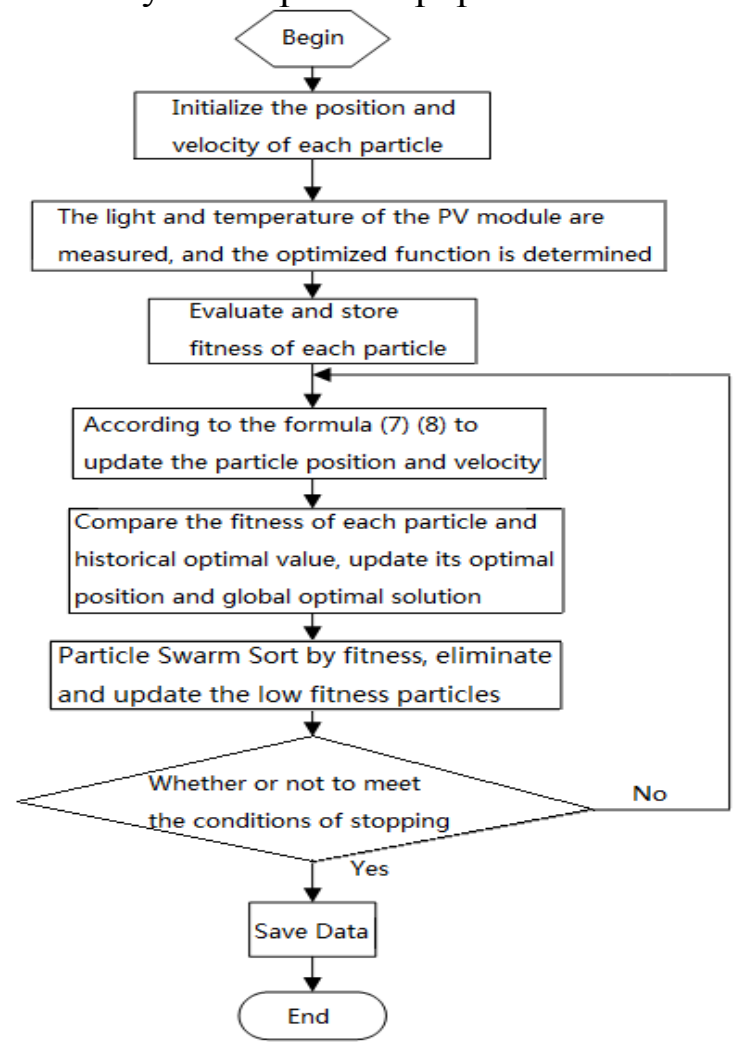

Fig.2 The flow chart of improved PSO

\section{Analysis based on matlab}

According to the analysis of algorithms above, modeling simulation comparison is made between traditional PSO and which improved by natural selection. Assuming the standard test temperature $\left(25^{\circ} \mathrm{C}\right)$, the short-circuit current of PV modules in the standard light intensity $\left(1000 \mathrm{~W} / \mathrm{m}^{2}\right)$ is $7 \mathrm{~A}$, the open-circuit voltage is $0.6 \mathrm{~V}$. Centralized PV array is given uneven illumination in the simulation, and its light conditions are as shown in the table 1 and table 2 below. Five PV arrays are connected in series to each other in the experiment, and each array contains 10 PV modules in series, and all the arrays are in the constant and same test temperature.

Table1. Array illumination conditions in experiment one

\begin{tabular}{lccccc}
\hline Array & 1 & 2 & 3 & 4 & 5 \\
\hline $\begin{array}{l}\text { Light intensity } \\
\text { density } / \mathrm{w} / \mathrm{m}^{2}\end{array}$ & 3000 & 2250 & 1500 & 1000 & 750 \\
\hline
\end{tabular}

Table2. Array illumination conditions in experiment two

\begin{tabular}{cccccc}
\hline Array & 1 & 2 & 3 & 4 & 5 \\
\hline $\begin{array}{c}\text { Light intensity } \\
\text { density } / w / m^{2}\end{array}$ & 3000 & 2700 & 2000 & 1200 & 300 \\
\hline
\end{tabular}


Track the maximum power point of centralized photovoltaic array using these two algorithms separately. In the experiments, take the size of the particle population as 50 , the maximum number of iterations is 60 , the learning factors of particles $c_{1}$ and $c_{2}$ are both 2 and the inertia weight is 0.9 . The tracking results of the two ways in two simulations are shown as follows:

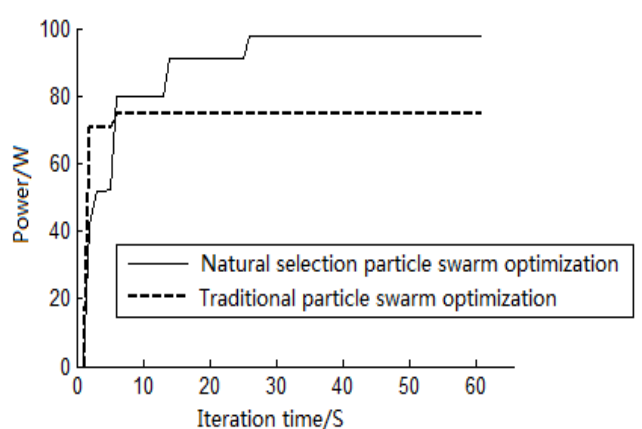

Fig.3 The effect of two MPPT methods in the first experiment

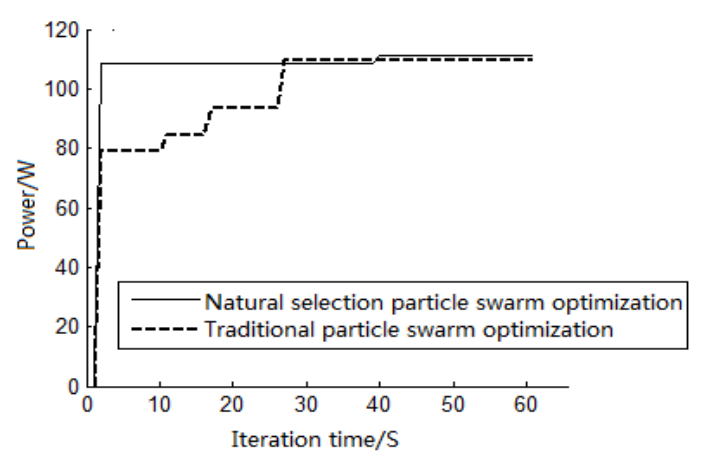

Fig.4 The effect of two MPPT methods in the second experiment

As shown in Fig.3, the improved PSO based on natural selection can quickly jump out after falling into local extreme point and can find the global optimization of MPPT finally, while traditional PSO algorithm will result in premature convergence and fall into local extreme points. In Fig.4, both of the algorithms can finally find the optimization of centralized arrays, but the improved solved faster, and the output characteristic of power is more stable, which prove that photovoltaic array has better optimization speed and solution stability in the improved MPPT algorithm.

\section{Summary}

In non-uniform illumination conditions, the new method which combine the mechanism of natural selection with traditional MPPT PSO, and simulation comparisons are made between the new and traditional particle swarm algorithm through MATLAB. As shown in the simulation results, the improved method can find the maximum power point of the PV array in complex lighting conditions faster and more stably than traditional algorithms, and can avoid premature convergence better. In addition, this method not only applies to non-uniform illumination conditions, but also applies to general uniform lighting conditions. The new method has good universality.

\section{References}

[1] Carrasco J M, Franquelo L G, Bialasiewicz J T, et al. Power-electronic systems for the grid integration of renewable energy sources: a survey[J].IEEE Transactions on Industrial Electronics, 2006, 53(4):1002-1016.

[2] Masafumi Mayatake, Mummadi Veerachary, Fuhito Toriumi,et al.Maximum Power Point Tracking of Multiple Photovoltaic Arrays: A Particle Swarm Optimization Approach[J].IEEE Transactions on Aerospaceand Electronic Systems,2011,47(1):367-380.

[3] Kashif Ishaque, Zainal Salam, Muhammad Amjad, et al.An Improved Particle Swarm Optimization(PSO)-Based MPPT for PV with Reduced Steady-State Oscillation[J].IEEE Transactions on Power Electronics,2012,27(8):3627-3638.

[4]Patel H, Agarwal V. MATLAB-based modeling to study the effects of partial shading on PV array characteristics [J].IEEE Transactions on Energy Conversion,2008,23(1):302-310. 